\title{
$M$-GROUPS WITH SYLOW TOWERS
}

\author{
ELSA L. GUNTER
}

(Communicated by Warren J. Wong)

\begin{abstract}
Let $G$ be a finite group, all of whose irreducible complex characters are induced from linear characters. Suppose that $G$ has a normal series of Hall subgroups $G_{i} \triangleleft G$ such that $G_{0}=1, G_{n}=G$, and $\left|G_{i}: G_{i-1}\right|$ is a power of a prime, for each $i=1, \ldots, n$. If $N$ is a normal subgroup of $G$, then every irreducible complex character of $N$ is induced from a linear character.
\end{abstract}

\section{INTRODUCTION}

A finite group is said to be an $M$-group if each of its irreducible characters can be induced from a linear character of some subgroup. In [3], Dornhoff showed that normal Hall subgroups of $M$-groups were $M$-groups, and asked if normal subgroups of $M$-groups were again $M$-groups. In [1], Dade gave an example of an $M$-group which had a normal subgroup which was not an $M$ group. Dade's example, however, depended very strongly on the use of the prime 2. Therefore, the question of whether normal subgroups of odd $M$-groups were again $M$-groups was left open, and remains so to the present.

The most straightforward approach to the problem of normal subgroups is to try to work "one character at a time". That is to say, try to prove that whenever a monomial character is restricted to a normal subgroup the irreducible constituents of the restriction are also monomial characters. Unfortunately, this approach does not work. Berger has given an example (see [5], Example 6.4) of a group which is the semidirect product of an extra-special 5-group by an extra-special 3-group, and which has a monomial character whose restriction to a normal subgroup has an irreducible constituent which is not monomial. This group, of course, is not an $M$-group. In fact, in this paper it will be shown that no $M$-group with such a simple structure can be a counterexample to the question of whether normal subgroups of $M$-groups are again $M$-groups. Namely, the following is shown:

Theorem. Let $G$ be an $M$-group with a Sylow tower. Then every normal subgroup of $G$ is again an $M$-group.

Received by the editors March 7, 1988 and, in revised form, August 8, 1988.

1980 Mathematics Subject Classification (1985 Revision). Primary 20C15, $20 \mathrm{D} 10$.

Research done under the supervision of I. M. Isaacs, University of Wisconsin, Madison. 


\section{INDUCTORS}

The difficulty in trying to determine whether or not a given character of a group is monomial is that in general there is no set way of knowing where to find a subgroup with a linear character to induce to the given character. Inductors provide us with subgroups, many of whose irreducible characters induce irreducibly. When we can conclude that these irreducible characters are themselves induced in the same manner as the characters that they are inducing, then we have a means of searching for an appropriate subgroup to induce our original monomial character.

1. Definition. We shall call $(G, N, \theta)$ a character trio if $N \triangleleft G$ and $\theta \in \operatorname{Irr}(N)$. If, in addition, $\theta$ is invariant in $G$, we shall say that $(G, N, \theta)$ is a character triple. Let $(G, N, \theta)$ be a character trio and let $G_{\theta}$ be the inertia subgroup of $\theta$ in $G$. Let $H \leq G_{\theta}$ and $(H, M, \varphi)$ be a character triple. Then the triple $(H, M, \varphi)$ is called an inductor for the trio $(G, N, \theta)$ if $G_{\theta}=H N$, if $M=H \cap N$, and if $\theta=\varphi^{N}$. Pictorially, we have:

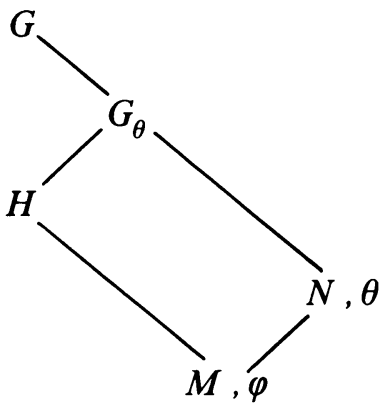

The notions of character triple and inductor can both be found in the work of Isaacs (see [7]). The idea of an inductor is similar to the idea of a search introduced by Parks in [8], and is used here to much the same purpose.

Note that an inductor for the character trio $(G, N, \theta)$ is the same thing as an inductor for the character triple $\left(G_{\theta}, N, \theta\right)$. In particular, $\left(G_{\theta}, N, \theta\right)$ is an inductor for $(G, N, \theta)$. Also note that if $(H, M, \varphi)$ is an inductor for $(G, N, \theta)$ and $(T, K, \psi)$ is an inductor for $(H, M, \varphi)$, then $(T, K, \psi)$ is an inductor for $(G, N, \theta)$.

The name inductor is justified by the following lemma.

2. Lemma. Let $(G, N, \theta)$ be a character trio and let $(H, N, \varphi)$ be an inductor for $(G, N, \theta)$. The character induction affords a bijection between the set of irreducible constituents of $\varphi^{H}$ and the set of irreducible constituents of $\theta^{G}$.

Proof. This is just Corollary 4.3 of [6] combined with Theorem 6.11 of [4].

One of the most important ways of producing inductors is through inertia subgroups. The following lemma is evident in the proof of Theorem 7.1 in [2]. A proof is included here for the sake of completeness. 
3. Lemma. Let $H$ be a finite group and let $A$ and $M$ be normal subgroups of $H$ with $A \leq M$. Let $\varphi$ be an irreducible character of $M$ which is invariant in $H$ and let $\lambda$ be an irreducible constituent of $\varphi_{A}$. If $H_{\lambda}$ and $M_{\lambda}$ are the inertia subgroups for $\lambda$ in $H$ and $M$, respectively, and if $\varphi_{\lambda}$ is the Clifford correspondent for $\varphi$ with respect to $\lambda$, then $\left(H_{\lambda}, M_{\lambda}, \varphi_{\lambda}\right)$ is an inductor for $(H, M, \varphi)$.

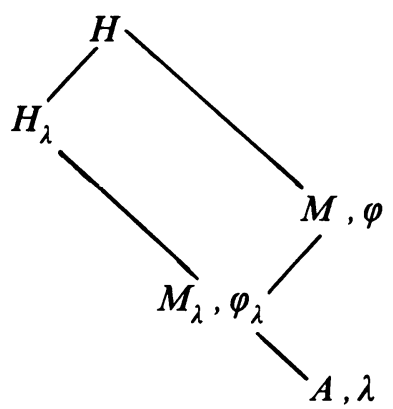

Proof. Since $\varphi$ is invariant in $H$, the $H$-conjugates of $\lambda$ are precisely the irreducible constituents of $\varphi_{A}$, which are precisely the $M$-conjugates of $\lambda$. Therefore, by the same argument as that for the Frattini argument, we have that $H=M H_{\lambda}$. That $M_{\lambda}=M \cap H_{\lambda}$ follows immediately from the definitions of $M_{\lambda}$ and $H_{\lambda}$. That $\left(\varphi_{\lambda}\right)^{M}=\varphi$ is given to us by Clifford's Theorem (see Theorem 6.11 of [4]).

Let $(G, N, \theta)$ be a character trio and suppose we want to show that all the characters lying over $\theta$ are monomial. When arguing by induction it would be useful to know when we can find a well-behaved inductor $(H, M, \varphi)$ for $(G, N, \theta)$ such that, if $\psi \in \operatorname{Irr}(H)$ which lies over $\varphi$ and if $\psi^{G}=\chi \in \operatorname{Irr}(G)$, then $\psi$ is monomial whenever $\chi$ is. For if we have such conditions, then Lemma 2 tells us that we may drop down to the well-behaved character triple $(H, M, \varphi)$.

4. Definition. Let $(G, N, \theta)$ be a character trio and let $(H, M, \varphi)$ be an inductor for $(G, N, \theta)$. Then we shall call $(H, M, \varphi)$ a monomial-preserving inductor for $(G, N, \theta)$ if every irreducible constituent, $\psi$, of $\varphi^{H}$ is monomial whenever $\psi^{G} \in \operatorname{Irr}(G)$ is. We shall call $(H, M, \varphi)$ a linear inductor for $(G, N, \theta)$ if $\varphi(1)=1$.

As we saw with Lemma 3, inertia subgroups give us a way of generating inductors, but we need to know when we can conclude that the inductors so obtained are monomial preserving. (They need not be in general.) The next two lemmas give us some conditions under which we may draw such conclusions.

5. Lemma. Let $H$ be a finite group, let $A$ be a normal subgroup of $G$, and let $\lambda$ be a linear character of $A$. Then $\left(H_{\lambda}, A, \lambda\right)$ is a monomial-preserving inductor for $(H, A, \lambda)$.

Proof. This is an easy consequence of Lemma 4.1 of [2]. 
6. Lemma. Let $Q$ be a normal nilpotent subgroup of $G$, and let $\theta$ be an irreducible character of $Q$. If $G_{\theta}$ is the inertia subgroup for $\theta$ in $G$, then $\left(G_{\theta}, Q, \theta\right)$ is a monomial-preserving inductor for $(G, Q, \theta)$.

Proof. This is directly implied by Theorem 3.1 of [8].

Earlier it was stated that if $(H, M, \varphi)$ were a well-behaved monomialpreserving inductor for $(G, N, \theta)$ then by using induction on the group's order, we could reduce to considering the case of $G=H$. The type of good behavior that it will turn out we need is for all of the Abelian subgroups of $M / \operatorname{ker} \varphi$ which are normal in $H / \operatorname{ker} \varphi$ to be under tight control.

7. Definition. Let $N$ be a normal subgroup of $G$. Suppose that for every subgroup $A \leq N$, if $A$ is Abelian and $A$ is normal in $G$, then $A$ is cyclic and $A \leq \mathbf{Z}(G)$. Then $N$ is said to be well situated in $G$.

8. Lemma. Let $Q$ be a nilpotent normal subgroup of $G$ and let $\theta \in \operatorname{Irr}(Q)$. Then there exists a monomial-preserving inductor $(H, M, \varphi)$ for $(G, Q, \theta)$ with $M / \operatorname{ker} \varphi$ well situated in $H / \operatorname{ker} \varphi$.

Proof. By Lemma 6 we have that $\left(G_{\theta}, Q, \theta\right)$ is a monomial-preserving inductor for $(G, Q, \theta)$. Among those inductors for $(G, Q, \theta)$ which are monomialpreserving choose an inductor $(H, M, \varphi)$ with $|H|$ minimal. Suppose that $M / \operatorname{ker} \varphi$ is not well situated in $H / \operatorname{ker} \varphi$.

Let $A$ be a normal subgroup of $H$ which is contained in $M$, and contains $\operatorname{ker} \varphi$. Suppose that $A / \operatorname{ker} \varphi$ is Abelian. Then every irreducible constituent of $\varphi_{A}$ is linear. Let $\lambda$ be one such. Since $\varphi$ is $H$-invariant, $A / \operatorname{ker} \varphi$ is cyclic and central in $H / \operatorname{ker} \varphi$ iff $\lambda$ is invariant in $H$. Therefore, since $M / \operatorname{ker} \varphi$ is not well situated in $H$, there exists $A \leq M$ with $A \triangleleft H$ and there exists an irreducible constituent $\lambda$ of $\varphi_{A}$ with $\lambda(1)=1$, but such that $\lambda$ is not invariant in $H$.

Since $\varphi$ is invariant in $H$, by Lemma 3 we have that $\left(H_{\lambda}, M_{\lambda}, \varphi_{\lambda}\right)$ is an inductor for $(H, M, \varphi)$. In addition, by Lemma 5 , since $\lambda(1)=1$, we have that $\left(H_{\lambda}, A, \lambda\right)$ is a monomial-preserving inductor for $(H, A, \lambda)$. Therefore, every irreducible constituent $\psi$ of $\lambda^{H_{\lambda}}$ is monomial whenever $\psi^{H}$ is. Now, $\varphi_{\lambda}$ is an irreducible constituent of $\lambda^{M_{\lambda}}$, so every irreducible constituent of $\left(\varphi_{\lambda}\right)^{H_{\lambda}}$ is an irreducible constituent $\lambda^{H_{\lambda}}$. Hence, $\left(H_{\lambda}, M_{\lambda}, \varphi_{\lambda}\right)$ is a monomialpreserving inductor for $(H, M, \varphi)$. However, a monomial-preserving inductor for a monomial-preserving inductor for $(G, Q, \theta)$ is a monomial-preserving inductor for $(G, Q, \theta)$. Since $H_{\lambda}<H$, this contradicts the minimality of $|H|$.

This next result will be a key step in the proof that all subnormal subgroups of an $M$-group with a Sylow tower are $M$-groups.

9. Lemma. Let $Q$ be a normal Hall $\pi$-subgroup of $G$ and suppose that $Q$ is nilpotent. Let $\theta \in \operatorname{Irr}(Q)$ and suppose that every irreducible constituent of 
$\theta^{G}$ is monomial. Then there exists a linear monomial-preserving inductor for $(G, Q, \theta)$.

Proof. The proof is by induction on $|G|$. By Lemma 8 there exists a monomialpreserving inductor $(H, M, \varphi)$ for $(G, Q, \theta)$ such that $M / \operatorname{ker} \varphi$ is well situated in $H / \operatorname{ker} \varphi$. Since $(H, M, \varphi)$ is an inductor for $(G, Q, \theta)$, we have that $M=H \cap Q$. Therefore, $M$ is a normal Hall subgroup of $H$ since $Q$ is a normal Hall subgroup of $G$. Since $(H, M, \varphi)$ is a monomial-preserving inductor for $(G, Q, \theta)$ and every irreducible constituent of $\theta^{G}$ is monomial, by Lemma 2 every irreducible constituent of $\varphi^{H}$ is monomial. Therefore, since a linear monomial inductor for $(H, M, \varphi)$ is a linear monomial inductor for $(G, Q, \theta)$, by the inductive hypothesis on $|G|$ we may assume that $(G, Q, \theta)=(H, M, \varphi)$. Then, $\theta$ is invariant in $G$ and $Q / \operatorname{ker} \theta$ is well situated in $G / \operatorname{ker} \theta$. Since $\theta$ is invariant in $G$, we have that $\operatorname{ker} \theta \leq \operatorname{ker} \chi$ for every $\chi$ an irreducible constituent of $\theta^{G}$. Therefore, again by the inductive hypothesis, we may assume that $\operatorname{ker} \theta=1$. That is, we can assume that $\theta$ is faithful and $G$-invariant, and that $Q$ is well situated in $G$.

Since $Q$ is well situated in $G$, every characteristic Abelian subgroup of $Q$ must be contained in the center of $Q$. Since $Q$ is nilpotent, this implies that $Q$ has nilpotence class at most 2. That is, $Q / Z(Q)$ is Abelian. Our goal is to show that $Q$ is Abelian.

Since $\theta$ is invariant in $G$ and $Q$ is a Hall subgroup of $G$, we have that $\theta$ extends to $G$ (see Corollary 8.16 in [4]). Let $\chi$ be such an extension. Then $\chi$ is an irreducible constituent of $\theta^{G}$, and hence is monomial. Therefore, there exists $L \leq G$ and $\lambda \in \operatorname{Irr}(L)$ such that $\lambda(1)=1$ and $\lambda^{G}=\chi$. Now, $|G: L|=\chi(1)=\theta(1)$ is a $\pi$-number and $|G: Q|$ is a $\pi^{\prime}$-number. Therefore, $G=L Q$. In addition, $\left(\lambda_{L \cap Q}\right)^{Q}=\left(\lambda^{G}\right)_{Q}=\chi_{Q}=\theta$.

Let $A=L \cap Q$. Since $\left(\lambda_{A}\right)^{Q}=\theta$, it follows that $\mathbf{Z}(Q) \leq A$. Therefore, since $Q / \mathbf{Z}(Q)$ is Abelian, $A \triangleleft Q$. Moreover, since $Q \triangleleft G$, we have that $A=L \cap Q \triangleleft L$, and hence $A \triangleleft L Q=G$. Now, $\lambda$ is an irreducible constituent of $\theta_{A}$ and $\lambda$ is linear. Therefore, since $\theta$ is faithful, it follows that $A$ is Abelian. However, since $Q$ is well situated in $G$, we must have $A \leq \mathbf{Z}(Q)$. Since $\left(\lambda_{A}\right)^{Q} \in \operatorname{Irr}(Q)$, the only way that we can have $A \leq \mathbf{Z}(Q)$ is if $A=\mathbf{Z}(Q)=Q$.

\section{ConClusion}

10. Theorem. Let $G$ be a group with a Sylow tower. Let $N$ be a subnormal subgroup of $G$. Suppose that there exists a normal Hall subgroup $Q$ of $G$ such that $Q \leq N$ and such that $Q$ is nilpotent. Further suppose there exists $\theta \in \operatorname{Irr}(Q)$ such that every irreducible constituent of $\theta^{G}$ is monomial. Then every irreducible constituent of $\theta^{N}$ is monomial.

Proof. We shall proceed by induction first on $|G|$, second on $|G: Q|$, and third on $|G: N|$. Suppose that $G$ is a counterexample. By the inductive hypothesis on $|G|$ we must have that $\operatorname{core}_{G}(\operatorname{ker} \theta)=1$. 
Since $Q$ is a normal Hall subgroup of $G$ which is nilpotent and every irreducible constituent of $\theta^{G}$ is monomial, by Lemma 9 there exists a linear monomial-preserving inductor, $(H, T, \lambda)$, for $(G, Q, \theta)$. Now, since $(H, T, \lambda)$ is an inductor for $(G, Q, \theta)$, we have that $H Q=G_{\theta}$, that $T=$ $H \cap Q$, and that $\lambda^{Q}=\theta$. Since $Q$ is a normal Hall subgroup of $G$, we have that $T$ is a normal Hall subgroup of $H$. Let $K=H \cap N$. Then $K$ is a subnormal subgroup of $H$ containing $T$ since $N$ is a subnormal subgroup of $G$ containing $Q$. Pictorially, we have:

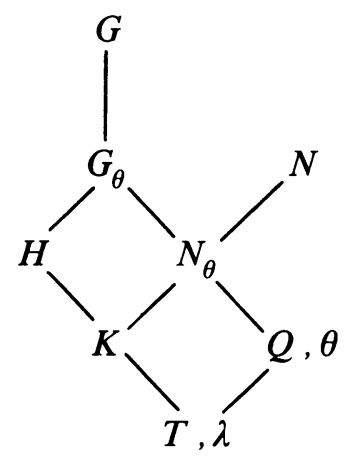

By Lemma 2 ever irreducible constituent of $\lambda^{H}$ induces to an irreducible constituent of $\theta^{G}$. Therefore, since every irreducible constituent of $\theta^{G}$ is monomial, and $(H, T, \lambda)$ is monomial preserving, every irreducible constituent of $\lambda^{H}$ is monomial. Assume that $H<G$. Then, by the inductive hypothesis on $|G|$, with $H$ in place of $G$, with $K$ in place of $N$, with $T$ in place of $Q$, and with $\lambda$ in place of $\theta$, we have that every irreducible constituent of $\lambda^{K}$ is monomial. Since $(H, T, \lambda)$ is an inductor for $(G, Q, \theta)$, we have that $\lambda$ is invariant in $H$ and hence in $K$, that

$$
K Q=(H \cap N) Q=H Q \cap N=G_{\theta} \cap N=N_{\theta},
$$

that

$$
K \cap Q=H \cap N \cap Q=H \cap Q=T \text {, }
$$

and that $\lambda^{Q}=\theta$. Therefore, $(K, T, \lambda)$ is an inductor for $(N, Q, \theta)$. Applying Lemma 2 to $(N, Q, \theta)$ and $(K, T, \lambda)$ we have that every irreducible constituent of $\theta^{N}$ is induced from an irreducible constituent of $\lambda^{K}$. However, since every irreducible constituent of $\lambda^{K}$ is monomial, this implies that every irreducible constituent of $\theta^{N}$ is monomial, contradicting that $G$ is a counterexample. Thus, we have that $G=H$, and therefore, $\theta$ is linear and invariant in $G$. Since $\theta$ is also faithful, we have that $Q \leq \mathbf{Z}(G)$.

Let $R$ be a normal Hall subgroup of $G$ such that $Q<R$ and $|R: Q|$ is a power of a prime, $p$. (We can find such an $R$ since $Q$ is a normal Hall subgroup of $G$ and $G$ has a Sylow tower.) Note that $R$ is nilpotent since $Q \leq \mathbf{Z}(G)$. Let $\varphi$ be an irreducible constituent of $\theta^{R}$. Then every irreducible constituent of $\varphi^{G}$ is an irreducible constituent of $\theta^{G}$, and hence 
every irreducible constituent of $\varphi^{G}$ is monomial. By the inductive hypothesis on $|G: Q|$, if $R \leq N$, then every irreducible constituent of $\varphi^{N}$ is monomial. Since this is true for every irreducible constituent of $\theta^{R}$ and since every irreducible constituent of $\theta^{N}$ lies over some irreducible constituent of $\theta^{R}$, we have that every irreducible constituent of $\theta^{N}$ is monomial. Therefore, we must have that $R \Varangle N$.

Now, $R$ is a normal subgroup of $G$, so $N R$ is a subnormal subgroup of $G$ since $N$ is. Therefore, since $N R>N$, by the inductive hypothesis on $|G: N|$, with $N R$ in place of $N$, we have that every irreducible constituent of $\theta^{N R}$ is monomial. If $N R<G$, then by the inductive hypothesis on $|G|$, this time with $N R$ in place of $G$, we would have that every irreducible constituent of $\theta^{N}$ is monomial. Therefore, we must have $G=N R$. Furthermore, since $Q \leq N \cap R$, we must have that $|G: N|=|N R: N|$ divides $|R: Q|$ and hence is a power of $p$.

If $N<M<G$ with $M \triangleleft G$, then, by the inductive hypothesis on $|G: N|$ with $M$ in place of $N$, every irreducible constituent of $\theta^{M}$ is monomial. Then, by the inductive hypothesis on $|G|$, using $M$ in place of $G$, we have that every irreducible constituent of $\theta^{N}$ is monomial. Therefore, we must have that $N$ is maximal normal in $G$ and $|G: N|=p$.

Let $\eta$ be an irreducible constituent of $\theta^{N}$ which is not monomial. Let $S=N \cap R$ and let $\psi$ be an irreducible constituent of $\eta_{S}$. Since $S \leq R$, we have that $S$ is nilpotent. Also, since $N$ and $R$ are both normal subgroups of $G$, so is $S$. Note that $\theta$ is an irreducible constituent of $\psi_{Q}$ and hence that every irreducible constituent of $\psi^{G}$ is an irreducible constituent of $\theta^{G}$, and thus is monomial.

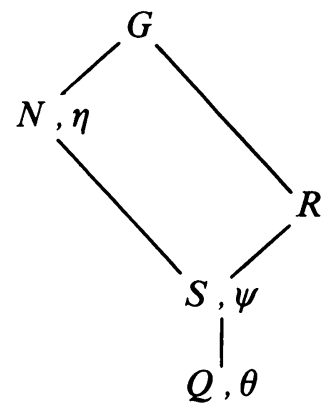

Consider $G_{\psi}$, the inertia subgroup for $\psi$ in $G$. Since $S$ is nilpotent, by Lemma 6 we have that $\left(G_{\psi}, S, \psi\right)$ is a monomial-preserving inductor for $(G, S, \psi)$. Therefore, since every irreducible constituent of $\psi^{G}$ is monomial, every irreducible constituent of $\psi^{G_{\psi}}$ is monomial. Suppose that $G_{\psi} \leq N$. Then $G_{\psi}$ is also the inertia subgroup for $\psi$ in $N$, and every irreducible constituent of $\psi^{N}$ is induced from an irreducible constituent of $\psi^{G_{\psi}}$. However, since every irreducible constituent of $\psi^{G_{\psi}}$ is monomial, this implies that every irreducible constituent of $\psi^{N}$, and in particular $\eta$, is monomial. Thus, we 
must have that $G_{\psi} \not N$. However, since $N$ is a maximal normal subgroup of $G$, we must have $G=N G_{\psi}$.

Now, $S \leq N \cap G_{\psi}$, so $\left|G: G_{\psi}\right|=\left|N G_{\psi}: G_{\psi}\right|=\left|N: N \cap G_{\psi}\right|$ which divides $|N: S|=|N: N \cap R|=|N R: R|=|G: R|$. Therefore, since $R$ is a normal Hall subgroup of $G$ we have that $R \leq G_{\psi}$. That is, we have that $\psi$ is invariant in $R$. Since $|R: S|=|G: N|=p$, it follows that every irreducible constituent of $\psi^{R}$ is an extension of $\psi$.

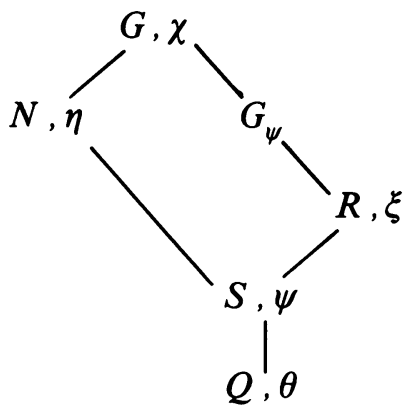

Let $\chi$ be an irreducible constituent of $\eta^{G}$ and let $\xi$ be an irreducible constituent of $\chi_{R}$ such that $\psi$ is an irreducible constituent of $\xi_{S}$. Then $\xi$ is an extension of $\psi$, and hence, $\psi(1)=\xi(1)$. Let $\pi=\pi(|R|)$. Then, since $R$ is a Hall $\pi$-subgroup of $G$, we have that the $\pi$-part of the degree of $\chi$ is $\chi(1)_{\pi}=\xi(1)$. Likewise, we have that $\eta(1)_{\pi}=\psi(1)$. Therefore, since $\psi(1)=\xi(1)$ and $|G: N|=p$ is a $\pi$-number and $\chi(1)$ divides $|G: N| \eta(1)$, it follows that $\chi(1)=\eta(1)$.

Now, $\chi$ is an irreducible constituent of $\theta^{G}$, and as such is monomial. Let $L \leq G$ and $\zeta \in \operatorname{Irr}(L)$ such that $\zeta(1)=1$ and $\zeta^{G}=\chi$. Since $\chi_{N}=\eta$, we have $L \Varangle N$. Therefore, $G=L N$ and

$$
\eta=\chi_{N}=\left(\zeta^{G}\right)_{N}=\left(\zeta_{L \cap N}\right)^{N}
$$

so $\eta$ is monomial.

11. Corollary. Let $G$ be an $M$-group with a Sylow tower. Then every subnormal subgroup of $G$ is an $M$-group.

Proof. Let $Q=1$ and $\theta=1$ in Theorem 10.

\section{REFERENCES}

1. E. C. Dade, Normal subgroups of M-groups need not be M-groups, Math. Z. 133 (1973), 313-317.

2. Monomial characters and normal subgroups, Math. Z. 152 (1981), 401-420.

3. L. Dornhoff, M-groups and 2-groups, Math. Z. 100 (1967), 226-256.

4. I. M. Isaacs, Character theory of finite groups, Academic Press, New York, 1976.

5. __ Primitive characters, normal subgroups, and M-groups, Math. Z. 177 (1981), 267-284.

6. _ Characters of $\pi$-separable groups, J. Algebra 86 (1984), 98-128. 
7. __ Character stabilizer limits relative to a normal nilpotent subgroup, J. Algebra 102 (1986), 367-375.

8. A. E. Parks, Nilpotent by supersolvable M-groups, Canad. J. Math. 37 (5) (1985), 934-962.

Department of Computer and Information Sciences, University of Pennsylvania, Philadelphia, PENNSYlVania 19104 